\title{
The dental pulp chamber evaluation by using cone-beam computed tomography
}

\author{
${ }^{* 1}$ Stella Samson, ${ }^{1}$ Viorel Nacu \\ ${ }^{1}$ Laboratory of Tissue Engineering and Cells Culture \\ Nicolae Testemitanu State University of Medicine and Pharmacy, Chisinau, the Republic of Moldova \\ Authors' ORCID iDs, academic degrees and contributions are available at the end of the article \\ ${ }^{*}$ Corresponding author: stelutzas@gmail.com \\ Manuscript received December 21, 2020; revised manuscript April 12, 2021; published online April 28, 2021
}

\begin{abstract}
Background: Cone-beam computed tomographic (СBCT) imaging is a valuable tool in dental practice. It is widely used in endodontic treatment for the root canal morphology examination. Therefore, the purpose of this study was to use CBCT to calculate the volume of the pulp chamber at different tooth groups.

Material and methods: This study conforms to protocols approved and in accordance with the ethics committee's requirements, informed consent was obtained from each patient. Morphologic measurements of 120 maxillary and 120 mandibular molars (from 40 patients, aged 18-45 years) were included in this study. СBCT images were taken using a Kodak 9500 (Dental Systems, Carestream Health) operated at $90 \mathrm{kVp}$ with a voxel size of $300 \mathrm{~mm}$ and a field of view of $90-50 \mathrm{~mm}$. All scans were taken following the manufacturer's recommendation protocol. According to the examination requirements, C-shaped roots, single-rooted molars, crowned teeth, and teeth with caries and/or restorations violating the pulp chamber were excluded. All measurements were taken on the coronal plane view.

Results: In the present study, we used CBCT imaging to gather information regarding pulp chamber volume. With the scanned 3-dimensional images, we were able to clinically determine the pulp chamber parameters using a standardized and defined spatial approach.

Conclusions: The data we collected here serve as a proof of principle for the analysis of dental landmarks before collecting stem cells. In this particular study, existing CBCT scans were used to provide useful information that can be used as a guide to determine the volume of the pulp chamber. Key words: stem cells, cone-beam computed tomographic imaging, pulp chamber.
\end{abstract}

Cite this article

Samson S, Nacu V. The dental pulp chamber evaluation by using cone-beam computed tomography. Mold Med J. 2021;64(2):49-52. https://doi.org/10.52418/ moldovan-med-j.64-2.21.09.

\section{Introduction}

Cone-beam computed tomography (CBCT) has become an indispensable method in dental practice. It is widely used in endodontic treatment to examine the morphology of root canals. CBCT can measure and calculate the volume of the pulp chamber, can provide more accurate information to predict the possible number of cells in the dental pulp.

Therefore, the purpose of this study was to use for the evaluation of the volume of the pulp chamber in different teeth groups.

Regenerative medicine based on the methodology of cell therapy and tissue engineering is a developing multidisciplinary field that involves biology, medicine and genetic manipulation. This type of therapy aims to maintain, restore or increase the function of tissues and organs, thus helping to treat human diseases of varying severity, from chronic to life-threatening situations [1]. In diseases that evolve with impaired tissue or organ function, stem cell research promises to provide us with a pathway to regenerative therapy. However, the application of this research in the treatment of human diseases is not possible without a thorough knowledge of the biological properties of all types of stem cells. In the context of a certain disease, it must first be decided which is the most appropriate type of stem cell: the embryonic one or adult type [2]. It is also necessary to identify a source of stem cells that is accessible and in large numbers and does not generate ethical concerns [3].

Possibly, the development of stem cells in dentistry will over time make a bigger revolution than implants. Metal alloys, composites and even titanium implants are not permanent solutions. Instead, stem cell technology will generate tissues that are compatible with the patients.

Stem cells are the cells that form the basis of the formation of all tissues and organs and are characterized by the ability to self-replicate and the ability to differentiate. Thus, from the stem cells in the pulp of temporary and permanent teeth, the substrate necessary for tooth formation can be generated. Teeth are an easily accessible source of harvesting postnatal stem cells from various tissues, including dental pulp, periodontal ligament, dental follicle, apical papilla of developing teeth [4].

Considerable progress has been made in recent years in understanding stem cell biology and new methods have been developed to obtain them and direct them to areas affected by the disease. The teeth have a complex structural composition that ensures both hardness and durability. 
However, this structure is vulnerable to trauma and bacterial infections [5]. When the tooth is damaged, but still in a condition that can be treated, regeneration of parts of the tooth structure can prevent or delay the loss of the entire tooth. This is of major importance, because the loss of teeth affects not only the basic functions of the stomatognathic apparatus, but also the quality of life [6]. The regenerative response of teeth to structural deterioration and degeneration is diverse because teeth are complex structures. Of all the dental structures, only the enamel is incapable of regeneration in its original structure, while the remaining tissues possess this quality, to varying degrees, depending on several factors [7]. Dental pulp plays an important role in tooth regeneration by participating in a process called restorative dentinogenesis. When the pulp tissue is exposed to a loss of dentin, direct styling therapy allows the pulp to form a new layer of dentin [8]. It has been observed that the use of various compounds, such as calcium hydroxide and mineral aggregate trioxide, promotes the activity of dentinogenesis [9]. Cells that remain in the healthy part of the pulp migrate to the affected part, proliferate due to growth factors released around the dentin matrix, and attach to the necrotic layer to form osteodentin [10]. Later, cells attached to osteodentin differentiate into odontoblasts to produce tubular dentin, thus forming repair dentin. This early mineralized tissue preserves the integrity of the pulp and serves as a protective barrier. When the tooth is still damaged, dental regeneration becomes difficult because it requires a healthy pulp [11]. Thus, larger traumas or advanced caries are treated by endodontic therapy, in which the entire pulp is cleaned and replaced with a canalicular filling material. However, live pulp is essential for maintaining homeostasis and tooth longevity [12]. An ideal form of therapy could be regeneration approaches in which the necrotic pulp is removed and replaced with regenerating pulpal tissues to revitalize the teeth [13]. In particular, regenerative pulp therapy would reconstitute normal continuous tissue at the pulp-dentin boundary by regulating tissue-specific processes of reparative dentinogenesis [14]. Two types of regeneration of the dental pulp can be considered depending on the clinical situation: partial in situ regeneration of the pulp or de novo synthesis of the pulp for its total replacement. Tissue engineering and regeneration of dental pulpal tissue remains a difficult task. A regenerated pulpal tissue must be functional and competent: it should be vascularized, contain cells similar to those of the natural pulp, be able to give birth to new odontoblasts, produce new dentin and be re-innervated. The first step for tissue engineering is to isolate cells with the correct phenotypes and propagate them in suitable culture media [15].

\section{Material and methods}

Initially to calculate the number of stem cells in the pulp chamber, we determined the volume of the pulp chamber. In this study, 120 upper molars and 120 lower molars were examined in 40 mature patients: 20 women and 20 men, aged between 18 and 45 years. The following study groups were formed: women aged 18-30 years, women aged 31-45, men aged 18-30, men aged 31-45. CBCT images were made using the Kodak 9500 (Dental Systems, Carestream Health) operated at $90 \mathrm{kVp}$, with a vox size of $300 \mu \mathrm{m}$ and a field of view of $90 \times 150 \mathrm{~mm}$. All scans were performed using the manufacturer's recommended protocol.

In accordance with the requirements of the Research Ethics Committee, the informed consent was obtained from each patient (protocol No 25 of 31.01.2013).

Criteria for excluding subjects in the study: according to the examination requirements, teeth with $\mathrm{C}$-shaped roots, single-root molars, decayed teeth or large restorations were excluded.

Criteria for inclusion of the subjects in the study: intact teeth without fillings with two and three roots were included.

All measurements were performed in the coronal plane. The appropriate selection of the section was made as follows. Initially the coronal plane of the molar was aligned according to the axial and sagittal point of view of the tooth. It was adjusted so that the coronal view represented a straight longitudinal section of the tooth from the cusp tip to the fork.

Once the coronal plane was identified from the axial point of view, 5 lines were placed at 5 different marks on the tooth (fig. 1, 2): L1: the beginning of the pulp chamber, L2: the floor of the pulp chamber, L3: the first point of separation between the roots (fork) and L4: the last point of separation between the roots (at the complete separation of the root), L5: the tip of the root.

All anatomical landmarks were approximated to plotted points, which were identified based on axial visualization. The slight difference that could have occurred in determining the points was negligible and did not affect the measurement. The volume of the pulp chamber was calculated according to the formula: $\mathrm{V}=\mathrm{h}{ }^{\star} \mathrm{L}^{\star} \mathrm{l}$, where $\mathrm{h}=\mathrm{A}+\mathrm{B}+$ $\mathrm{C}+\mathrm{D}$. Direct measurements were taken between the 5 lines and the following distances were calculated as follows.

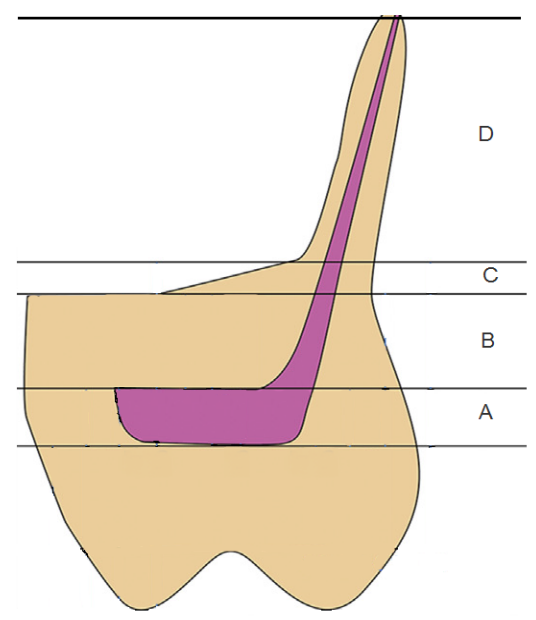

Fig. 1. Lines used as a benchmark for determining the volume of the pulp chamber

A - the beginning of the pulp chamber to the floor of the pulp chamber, $\mathrm{B}$ - the floor of the pulp chamber up to the first point of separation between the roots (fork), $\mathrm{C}$ - the first point of separation between the roots (the fork) until the last point of separation between the roots (at the complete separation of the root), D - the last point of separation between the roots (at the complete separation of the root) to the top of the root. 

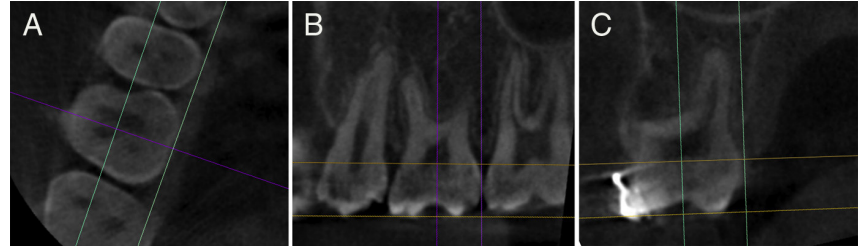

Fig. 2. СBCT images that identify the optimal view for measuring the volume of the pulp chamber of the wisdom tooth

A - axial view, B - sagittal vision, C - the green line of the sagittal plane passes along the longitudinal axis of the tooth. The yellow line of the axial plane passes perpendicular to the line of the sagittal plane and is parallel to the occlusal surface.

\section{Results}

We find that initially the indices from the experimental groups were identical, alternating, so that in the end the volume of the pulp chamber increased from the upper molar 3 to the upper molar 1, at the lower molars it inversely decreased from the lower molar 3 to the lower molar 1 (fig. 3). Currently, computed tomography offers us the possibility to automatically calculate on digital images (tomograms) the areas we are interested in. The data obtained show a direct correlation between the height, length, width of the pulp chamber and its volume.

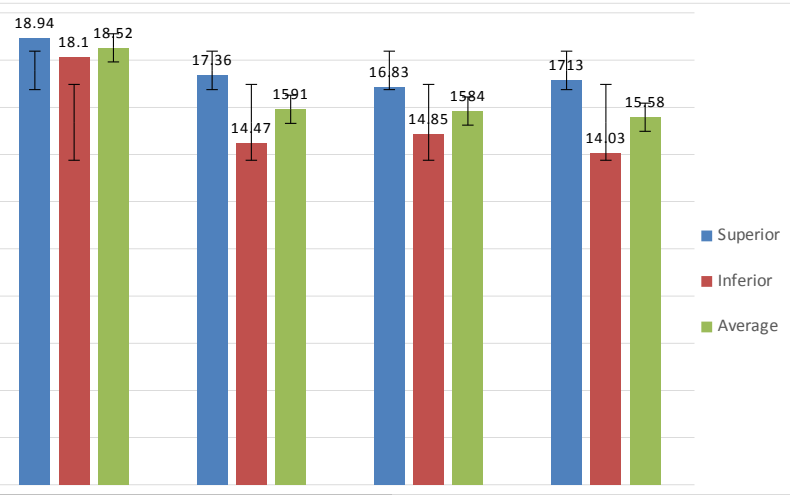

Fig. 3. The volume of the pulp chamber in the upper and lower molars in women and men

\section{Discussion}

This study investigated the relationship between the age and the volume of the pulp chamber of the upper and lower molars.

Sex and age are two major variants of estimating the volume of the pulp chamber, which was demonstrated in the present study. CBCT is an important tool for measuring the volume of the pulp chamber. In the present study, we used CBCT to gather information regarding pulp chamber landmarks relevant for volume determination.

Performing measurements of three-dimensional scanned images, allows determining the parameters of the pulp chamber using a defined standard of spatial approach. This study highlights the potential presence at different depths in the fork area and their identification using CBCT [16].

Selecting the optimal section for measuring the parameters was the critical part of this study. The coronal plan was used for the best view of the entire crown and the fork area. Adjustment of CBCT images from axial and sagittal vision allowed us to select the optimal coronal section to measure the parameters of the pulp chamber landmarks [17].

Using axial visualization as a guide allows us to accurately locate the desired parameters and prevented the overlapping of anatomical parameters in different planes. Such standardization would ensure the reproducibility of measurements [18].

In this study, we present 2 types of pulp chamber volume measurements at permanent molars: (1) measurements from the dental cusp tip, as in previous reports and (2) measurements from the central dental fossa. As access preparation is often initiated in the center of the occlusal surface, the central fossa appears to be a more appropriate reference point instead of the cusp tip used in previous studies. In addition, many variables can affect cusp height, such as location and size [19]. In previous studies, the bifurcation is always mentioned as a point where the tooth structure ends, and the roots separate. However, the fork is an area that results from the separation of two or more roots [10]. In this study, using axial vision, it was noted that the point of separation of the roots cannot always happen at the same level along the entire fork area. There were different bifurcation depths more common in the upper jaw (69\%) than the lower jaw (34\%), indicating that the fork is an area and that cannot be mentioned as a point [20]. Alignment of the scans in the center of the tooth can lead to overlapping anatomical landmarks and failure to identify the most coronal point where the roots separate. In this study, we identified the area of bifurcation at 2 different levels guided by axial vision. This allowed us to identify the different depth levels of the bifurcation area. Despite the wide variation in tooth length, the height of the crown appears to be very similar between all molars (8-9 $\mathrm{mm}$ from the central fossa and $10-11 \mathrm{~mm}$ from the tip of the tip). The distance from the central fossa to the bifurcation at the maxillary molars $(8.78-0.79 \mathrm{~mm})$ was very similar to that of the mandibular molars $(8.53-0.65 \mathrm{~mm})$. The variation is probably caused by the discrepancy in the cusp height between the molars of the upper and lower jaw. The height of the pulp chamber had the highest value for the maxillary molars $(38.2 \%)$ and $(44.4 \%)$ for the mandibular ones. These findings were similar to previous reports. Such a large variation is probably caused by the ongoing dentin deposition, which reduces the height of the pulp chamber. It can be interpreted that the calcification process appears on the ceiling of the pulp chamber as a protection mechanism against external stimuli. It has been reported by several authors that the reduction in pulp chamber height is the result of dentin deposition on the floor rather than on the ceiling [21].

It should be noted that such measurements and their differences cannot determine the location where the calcification process begins. Further investigations are needed to determine the effect of caries and restorations on the height of the pulp chamber relative to the central fossa [22].

In the present study, we focused only on whole teeth, without restorations or endodontic treatments to allow an adequate identification of the studied parameters. 
It seems that CBCT images, with those previously used methods, prove to be a useful and accurate tool to determine the parameters of the pulp chamber and which would allow an approximate assessment of the number of cells contained in the dental pulp.

\section{Conclusions}

1. The size of the pulp chambers according to gender showed that for three molars, the volume of the pulp chamber is $17.2 \pm \mathrm{mm}^{3}$ in women's and $17.88 \pm \mathrm{mm}^{3}$ in men's, the difference not being significant.

2. The determination of the volume of the pulp chamber, by non-invasive method, makes it possible to predict approximately the number of nucleated cells that can be obtained from a tooth until its extraction, thus excluding teeth with a lower cell potential.

3. It is necessary to assess the concordance between the volume of the dental chamber and the specific cell content in it and the concordance between the gender, the location of the tooth and the age of the donor.

\section{References}

1. Ciobanu PI, Lavrishcheva GI, Kozliuk AS. [Stimulation of osteogenesis by bone marrow cells in complicated fractures]. Chişinău: Stiinta; 1989. 181 p. Russian.

2. Friedman MS, Long MW, Hankenson KD. Osteogenic differentiation of human mesenchymal stem cells is regulated by bone morphogenetic protein-6. J Cell Biochem. 2006;98(3):538-554. doi: 10.1002/jcb.20719.

3. Esther P, Ferreira E, Andriamanalijaona R, et al. Hypoxia affects mesenchymal stromal cell osteogenic differentiation and angiogenic factor expression. Bone. 2007;40(4):1078-1087. doi: 10.1016/j.bone.2006.11.024.

4. Lieberman JR, Friedlaender GE. Bone regeneration and repair. Totowa: Humana press; 2005. 398 p.

5. Davies JE, editor. Bone engineering. Toronto: Em Squared Inc; 2000. $656 \mathrm{p}$.

6. Hing KA. Bone repair in the twenty-first century: biology, chemistry or engineering? Philos Trans A Math Phys Eng Sci. 2014;362(1825):28212850. doi: 10.1098/rsta.2004.1466.

7. Kaplan JC, Deplech M. Biologie moleculaire et medicine [Molecular biology and medicine]. 3rd ed. Paris: Flammarion Medecine-Sciences; 2007. 815 p. French.

8. Buttery LD, Bourne S, Xynos JD, et al. Differentiation of osteoblasts and in vitro bone formation from murine embryonic stem cells. Tissue Eng. 2001;7(1):89-99. doi: 10.1089/107632700300003323.

\section{Authors' ORCID iDs and academic degrees}

Stella Samson, MD, PhD Applicant - https://orcid.org/0000-0002-0030-9193

Viorel Nacu, MD, PhD, Professor - https://orcid.org/0000-0003-2274-9912

\section{Authors' contribution}

SS designed the study, collected, processed, and interpreted the data and drafted the manuscript. NV designed the research and revised the manuscript critically. Both authors revised and approved the final version of the manuscript.

\section{Funding}

This study was supported by Nicolae Testemitanu State University of Medicine and Pharmacy, and the research project "Nanoarchitecture in base of $\mathrm{GaN}$ and tridimensional matrix from biologic material for application in microfluids and tissue engineering" - 20.80009.5007.20, offered by the National Agency for Research and Development of the Government of the Republic of Moldova. The authors are independent and take responsibility for the integrity of the data and accuracy of the data analysis.

\section{Ethics approval and consent to participate}

The research was approved by the Research Ethics Committee of Nicolae Testemițanu State University of Medicine and Pharmacy (protocol No $70 / 75$ of 21.05.2018).

\section{Conflict of Interests}

Nothing to disclose.
- Aubin JE Triffit JT Mesenchymal stem cells and osteoblast differentiation. In: Bilezikian JP, Raisz LG, Rodan GA, editors. Principles of bone biology. Vol. 1. San Diego: Academic Press; 2002. p. 59-81.

Tidmarsh BG. Micromorphology of pulp chambers in human molar tb00871.x.

11. Matherne RP, Angelopoulos C, Kulild JC, Tira D. Use of cone-beam 2008;34(1):87-89. doi: 10.1016/j.joen.2007.10.016.

Muthe Stanley HR, Matthews JB, et al. Age-related odontometric Endod. 2002;93(4):474-482. doi: 10.1067/moe.2002.120974.13. Karl M, Palarie V, Nacu V, Grobecker-Karl T. Pilot animal study aimed at assessing the mechanical quality of regenerated alveolar bone. Int J Oral Maxillof Implants. 2020;35(2):313-319. doi: 10.11607/jomi.7694.

14. Enneking WF, Campanacci DA. Retrieved human allografts: a clinicopathological study. J Bone Joint Surg Am. 2001;83(7):971-986.

15. Shaw L, Jones AD. Morphological considerations of the dental pulp chamber from radiographs of molar and premolar teeth. J Dent.

Braniste T, Cobzac V, Ababii P, Plesco I, Raevschi S, Didencu A, Maniuc M, Nacu V, Ababii I, Tiginianu I. The influence of semiconductor nanoparticles upon the activity of mesenchymal stem cells. In: TiginI, Sontea V, Railean S, editors. 4th International Conference on Nanotechnologies and Biomedical Engineering. IFMBE Proceedings. 2019. Vol. 77. Cham: Springer; 2019. p. 607-611. doi: 10.1007/978-3 030-31866-6_108.

17. Deans RJ, Moseley AB. Mesenchymal stem cells: biology and potential clinical uses. Exp Hematol. 2000;28(8):875-884. doi: 10.1016/s0301472x(00)00482-3.

Morse DR. Age-related changes of the dental pulp complex and their relationship to systemic aging. Oral Surg Oral Med Oral Pathol 1991;72(6):721-745. doi: 10.1016/0030-4220(91)90019-9.

. The relationship of the pulp chamber to doi: 10.1016/0022-3913(77)90168-8.

20. Plotino G, Tocci L, Grande NM, et al. Symmetry of root and root canal morphology of maxillary and mandibular molars in a white population: a cone-beam computed tomography study in vivo. J Endod. 2013;39(12):1545-1548. doi: 10.1016/j.joen.2013.09.012.

Silva EJ, Nejaim Y, Silva AV, et al. Evaluation of root canal configuration computed tomography: an in vivo study. J Endod. 2013;39(7):849-852. doi: 10.1016/j.joen.2013.04.030.

. Sterrett JD, Pelletier H, Russell CM. Tooth thickness at the furcation 10.1111/j.1600-051x.1996.tb00585.x. 1984;12(2):139-145. doi: 10.1016/0300-5712(84)90048-4. of mandibular molars in a Brazilian population by using cone-beam 